\title{
Efecto de la estacionalidad y lluvias en la avifauna del Chaco Serrano Austral, Argentina
}

\author{
Gustavo A. Fava ${ }^{1}$, Juan C. Acosta ${ }^{1,2} \&$ Graciela M. Blanco ${ }^{1,2}$
}

1. Centro de Investigaciones de la Geósfera y Biosfera (CIGEOBIO), CONICET - Facultad de Ciencias Exactas, Físicas y Naturales, Universidad Nacional de San Juan. CP: J5404DCS, San Juan, Argentina; gustavo.fava21@gmail.com

2. Departamento de Biología y Centro de Investigaciones de la Geósfera y Biosfera (CIGEOBIO), CONICET - Facultad de Ciencias Exactas, Físicas y Naturales, Universidad Nacional de San Juan. CP: J5404DCS, San Juan, Argentina; jcacostasanjuan@gmail.com, blancogracielam@gmail.com

Recibido 30-XI-2016. Corregido 23-III-2017. Aceptado 24-IV-2017.

\begin{abstract}
The effects of seasonality and precipitation in the avifauna of the Argentine Southern Chaco Serrano. In subtropical regions like the Chaco Serrano $\left(33^{\circ} \mathrm{S}\right)$, rainfall has a marked seasonality, and water flood, caused by heavy rains, may have important consequences for dependent ecosystems. Conversely, the high pressure on available resources during dry periods can also affect the fauna diversity and distribution. Even though birds have shown to be excellent ecological indicators of these shifts in the environment, currently little is known about the avifauna of the Argentine Chaco Serrano phytogeographic district. Our aim was to study the resident bird population status in the Southern Chaco Serrano, its fluctuations in richness and abundance, and its relationship to seasonal variations, in monthly and annual hydroperiods. In order to assess the resident bird status over a two year period (2011 Mar.-2013 Feb.) we carried out 18 sampling surveys during pre-breeding (spring), breeding (summer) and non breeding periods (autumn-winter), in the Northeastern part of the Valle Fértil Natural Park, San Juan province, Argentina. During each site survey, we recorded bird assemblages found in six transects, and obtained bird abundance and species diversity. A mixed general linear model was employed to evaluate relationships between the studied variables. We found a total of 98 species of birds belonging to 33 families (44 resident species, 23 spring visitors, six summer visitors, two winter visitors and 23 with scarce registers). We also registered 68 of these species in both hydroperiods, 28 only during rainy periods and only two during the dry period. Both richness and abundance showed the highest values during the pre-breeding and rainy seasons, while different tendencies were detected during the isolated monthly rainfalls. These changes in bird densities were associated with the arrival of migratory species, mainly spring visitors, and probably in relationship with their reproductive activity, and to seasonal fluctuations in food resources. The Southern Chaco Serrano is a subtropical semiarid place that seems to be an important breeding habitat for many migrant bird species, which in turn promoted seasonal changes in species density and diversity, mainly during rainy years. This work contributed to our understanding on the causes of richness and abundance variations on birds, and will contribute to this important group management and conservation in this area. Rev. Biol. Trop. 65 (3): 953-961. Epub 2017 September 01.
\end{abstract}

Key words: ornithology, Valle Fértil Natural Park, ecology, hydro periods, dry, semiarid.

Los ambientes subtropicales son marcadamente estacionales y en general dicha estacionalidad está asociada con los períodos de lluvias más que con las variaciones de la temperatura (Sarmiento, 1972; Huxman et al., 2005). En estos ambientes la vegetación crece y se reproduce en años lluviosos, mientras que durante los años de sequía, las plantas sufren estrés seguido por la abscisión de las hojas y el cese de su crecimiento (Brinson, 1993). Estas fluctuaciones afectan tanto la estructura del hábitat como la disponibilidad de los recursos, lo cual genera cambios notorios en la composición y abundancia de las comunidades de aves (Maldonado, 2012).

Las aves son consideradas como excelentes indicadores ecológicos y facilitan evaluar los cambios de su hábitat (Bibby, Jones 
\& Marsden, 1998; Milesi, Marone, Lopez de Casenave, Cueto \& Mezquida, 2002). Una de las formas de evaluar la influencia que ejercen las precipitaciones sobre la avifauna, es considerar el hidroperíodo como el nivel de agua acumulado en su hábitat, el tiempo que permanece disponible allí y en qué momento del año se producen las precipitaciones, como descriptor del periodo de acumulación de agua (Brinson, 2004; Ginzburg, Adámoli, Herrera \& Torrella, 2005). De este modo, hidroperíodos contrastantes, pueden reflejar cambios en la avifauna, donde algunas especies están adaptadas a condiciones de inundación o de sequía, mientras que otras persisten en ambas condiciones (Beltzer \& Neiff, 1992; Casco, Neiff $\&$ Neiff, 2005).

En estos ecosistemas, la composición de la avifauna también estaría determinada por el arribo o la partida de especies visitantes invernales, estivales o por desplazamientos locales que realizan en respuesta a la variabilidad de los recursos tróficos (Codesido \& Bilenca, 2004; Marateo \& Arturi, 2013). Estos desplazamientos pueden variar tanto en la distancia como en el momento de la migración y dependen principalmente de la calidad del hábitat (Jahn, Levey, Johnson, Mamani \& Davis, 2006; Buehler \& Piersma, 2008; Lourenço \& Piersma, 2015).

En el Chaco Serrano de Argentina existen varios registros documentados sobre los aspectos biogeográficos de la avifauna (Ochoa de Masramón, 1983; Nores \& Yzurieta, 1983; Olrog \& Capllonch, 1986; Capurro \& Bucher, 1988; Haene, Krapovickas, Moschione, \& Gómez, 1995; Nores, 1995; Cid \& Caviedes Vidal, 2005; Camperi, Darrieu \& Juárez, 2008). Sin embargo, poco se conoce sobre las especies que componen la comunidad de aves en su sector austral; hasta el momento, no se dispone de estudios acerca de la variación estacional en función del hidroperíodo. Frente a este panorama, los objetivos de este trabajo fueron (1) evaluar el estatus de residencia de aves en el margen austral del Chaco Serrano y (2) determinar la variabilidad de su riqueza y abundancia en función de la variación estacional, de los hidroperíodos mensuales y anuales.

\section{MATERIALES Y MÉTODOS}

Área de estudio: El estudio comprendió el sector austral del distrito del Chaco Serrano, en el Parque Natural Provincial Valle Fértil, entre La Majadita y Los Bretes (3043' S - 67²9' W), en la provincia de San Juan, Argentina. Salinas de Salmuni, Maldonado, Lund y Puigdengolas (com. pers.), en un estudio de delimitación de la erosión hídrica mediante el procesamiento digital de imágenes de Landsat $\mathrm{TM}$, encontraron que esta área está influenciada por una gran llanura aluvial.

$\mathrm{Su}$ flora tiene escasas modificaciones antrópicas dentro del desierto semiárido de la provincia (Márquez, Ripoll, Dalmaso, Ariza, \& Jordan, 2015) y son consideradas como prioritarias para la conservación (Herrera \& Ortiz, 2005). La misma es conformada por bosque de Schinopsis marginata como especie dominante, el Lithraea molleoides pobremente expandida en toda la sierra, y un matorral serrano formado principalmente por Acacia furcatispina, Prosopis torquata, Acacia aroma y Trichocereus terscheckii (Márquez et al., 2015).

Desde el punto de vista bioclimático, presenta un subclima semiárido, seco y con predominio de lluvia estivales (BShw, conforme a la clasificación de Köppen). La temperatura media anual es de $19.5^{\circ} \mathrm{C}$ con una mínima y máxima promedio entre 11 y $26^{\circ} \mathrm{C}$, respectivamente. La precipitación media anual registrada por el puesto meteorológico Valle Fértil, es de $358 \mathrm{~mm}$, con rango de medias entre 152 y 656 $\mathrm{mm}$ (datos proporcionados por el Departamento de Hidráulica de la Provincia de San Juan, Argentina, periodo 1970-2009).

La avifauna de la parte austral de este distrito, tanto al norte de nuestro sitio de estudio (Sierras de Famatina, de Soñogasta, de Peganzo y de la Morada; provincia de la Rioja) (Camperi et al., 2008), como hacia el sur: Sierras de las Quijadas y de San Luis; provincia de San Luis, y Sierras de Comechingones; provincia de Córdoba (Ochoa de Masramón, 1983; 
Nores \& Yzurieta, 1983; Cid \& Caviedes Vidal, 2005) son zonas destacadas como prioritarias para la conservación de la avifauna (Herrera \& Ortiz, 2005; Di Giacomo, De Francesco, \& Coconier, 2007).

Diseño de muestreo: En este estudio se realizaron 18 campañas de avistaje, entre marzo 2011 a febrero 2013. Los muestreos se realizaron con seis transectas de $6 \mathrm{~km}$ con un ancho fijo, de $60 \mathrm{~m}$ cada una, a lo largo de toda la matriz del paisaje. Los avistajes fueron realizados por dos personas a la vez, desde las 6 am a las 10 am y desde las $6 \mathrm{pm}$ a las $8 \mathrm{pm}$ en todos los muestreos, y estuvieron separados geográficamente por al menos $2 \mathrm{~km}$ entre sí.

El registro de la riqueza y abundancia de aves comprendió los individuos en tránsito como también los que buscan el alimento en vuelo (de las familias Cha, rapaces y golondrinas) y se utilizó Narosky y Yzurieta (2010) para su reconocimiento. Los registros fueron promediados en la estación de otoño-invierno (marzo 2011-2012, mayo 2011, junio 2012 y julio 2011-2012), de primavera (septiembre 2011-2012, agosto 2011, octubre 2012 y noviembre 2011-2012) y de verano (diciembre 2011-2012, enero 2011-2012 y febrero 2012-2013). Estos datos fueron usados para representar a la población de aves en los periodos no reproductivos, pre-reproductivos y reproductivos, respectivamente. Con el fin de establecer el estatus de residencia, cada especie fue clasificada de la siguiente manera: accidentales o dudosas, especies que por contar con un único registro, no pudieron ser incluidas en ninguna de las categorías anteriores; residentes, especies registradas durante todas las estaciones del año; visitantes invernales, especies únicamente registradas durante el periodo de otoño-invierno; visitantes estivales tempranos, especies registradas durante primavera-verano y visitantes estivales, especies registradas únicamente durante verano.

Para estimar el hidroperíodo mensual se calculó la lluvia acumulada en los 30 días anteriores al último día de cada muestreo. Para el hidroperíodo anual se consideró un año lluvioso con un valor de precipitación superior a la media esperada (con $415.2 \mathrm{~mm}$, desde marzo 2011 a febrero 2012), y un año de sequía con un valor de precipitación inferior al mínimo esperado (con $67.7 \mathrm{~mm}$, desde marzo 2012 a febrero 2013). Estos valores fueron estimados para la Sierra de Valle Fértil (30³9'24" $\mathrm{S}-67^{\circ} 36^{\prime} 12^{\prime \prime} \mathrm{W}$ ) por el puesto meteorológico más cercano en el Aeropuerto La Rioja (www. freemeteo.com.ar).

La variación de la riqueza y abundancia de aves en función del hidroperíodo mensual, del hidroperíodo anual (lluvioso y de sequía), de las estaciones (otoño-invierno, primavera y verano) y de las interacciones entre estas variables, fue analizada utilizando un modelo mixto para medidas repetidas (MMRM), utilizando el paquete de R "Ime4" (Bates, Maechler, Bolker, \& Walker, 2015) y las aproximaciones de Laplace para estimar los parámetros (Bolker et al., 2009). La variación generada entre cada muestreo fue incluida en el modelo como factor aleatorio.

Para definir la estructura óptima de cada modelo completo (es decir, con todos los efectos fijos y el aleatorio) se utilizó el criterio de información de Akaike (AIC) mediante análisis estadísticos de estimación de máxima verosimilitud restringida (REML), con la función "lmer" (Bates et al., 2015). La estructura de efectos fijos se simplificó mediante la eliminación de variables e interacciones que no fueron significativamente asociadas con la variable respuesta, de acuerdo a la prueba de Waldt (Bolker et al., 2009).

Estos análisis estadísticos se realizaron con el software R, versión 3.2.2 (The R Development Core Team, 2015). Para citar las especies de aves se siguió la listas taxonómica propuesta por el Comité de Clasificación de América del Sur (Remsen et al., 2016).

\section{RESULTADOS}

La abundancia de aves fue de 98 especies (51 paseriformes) pertenecientes a 34 familias, durante los dos hidroperíodos anuales. Las familias con mayor riqueza fueron: Tyrannidae 
(18 especies), Furnariidae (10 especies), Thraupidae y Columbidae (ambas con seis especies).

En ambos hidroperíodos fueron halladas 68 especies (el $69.4 \%$ del total) siendo las especies más abundantes: Zonotrichia capensis (Emberizidae), Myiopsitta monachus (Psittacidae), Patagioenas maculosa (Columbidae), Thectocercus acuticaudatus (Psittacidae) y Sicalis flaveola (Thraupidae). Asimismo, Elaenia parvirostris (Tyrannidae), Leptasthenura platensis (Furnariidae) y Knipolegus aterrimus (Tyrannidae) dominaron entre las 28 especies (el $28.6 \%$ del total) que sólo se registraron en el año lluvioso y Poospiza melanoleuca (Thraupidae) junto a Anairetes parulus (Tyrannidae), el $2 \%$ del total, sólo en el año de sequía.

Las variaciones de la riqueza de aves fue mejor explicada por el modelo que incluyó la variabilidad del hidroperíodo anual y de las estaciones, mientras que la interacción entre estas dos variables y el hidroperíodo mensual, no presentaron diferencias estadísticamente significativas (MMRM; $\mathrm{P}=0.90)$ (Fig. 1A). En el año lluvioso, la riqueza fue mayor respecto al de sequía (MMRM; $\mathrm{t}=3.71$; E.E. $=2.81 ; \mathrm{P}$ $=0.003$ ) (Fig. 1A). Respecto a las estaciones, esta variable fue mayor en verano (MMRM; $t$ $=5.66$; E.E. $=3.44 ; \mathrm{P}=0.001) \mathrm{y}$ en primavera $(\mathrm{MMRM} ; \mathrm{t}=4.21 ; \mathrm{E} . \mathrm{E} .=3.44 ; \mathrm{P}=0.002)$, respecto al otoño-invierno (Fig. 1A).

Por otro lado, las variaciones en la abundancia de especies fueron mejor explicadas por el modelo que incluyó la estacionalidad y sus interacciones con el hidroperíodo anual (Fig. 1B). Considerando las estaciones, la mayor abundancia fue en verano seguido de la primavera. Sin embargo, la abundancia de aves fue más baja en el año de sequía durante la primavera (MMRM; $\mathrm{t}=4.49$; E.E. $=42.03 ; \mathrm{P}=0.015$ ) $\mathrm{y}$ el verano $(\mathrm{MMRM} ; \mathrm{t}=7.12$; E.E.= 42.03; $\mathrm{P}$ $=0.001)$ (Fig. 1B). En contraste, la abundancia de aves en otoño-invierno no presentó diferencias estadísticamente significativas entre el año lluvioso y el año de sequía (MMRM; P $=0.58$ ) (Fig. 1B). La acumulación de lluvias mensuales no fue significativamente diferente (MMRM; P =0.895).
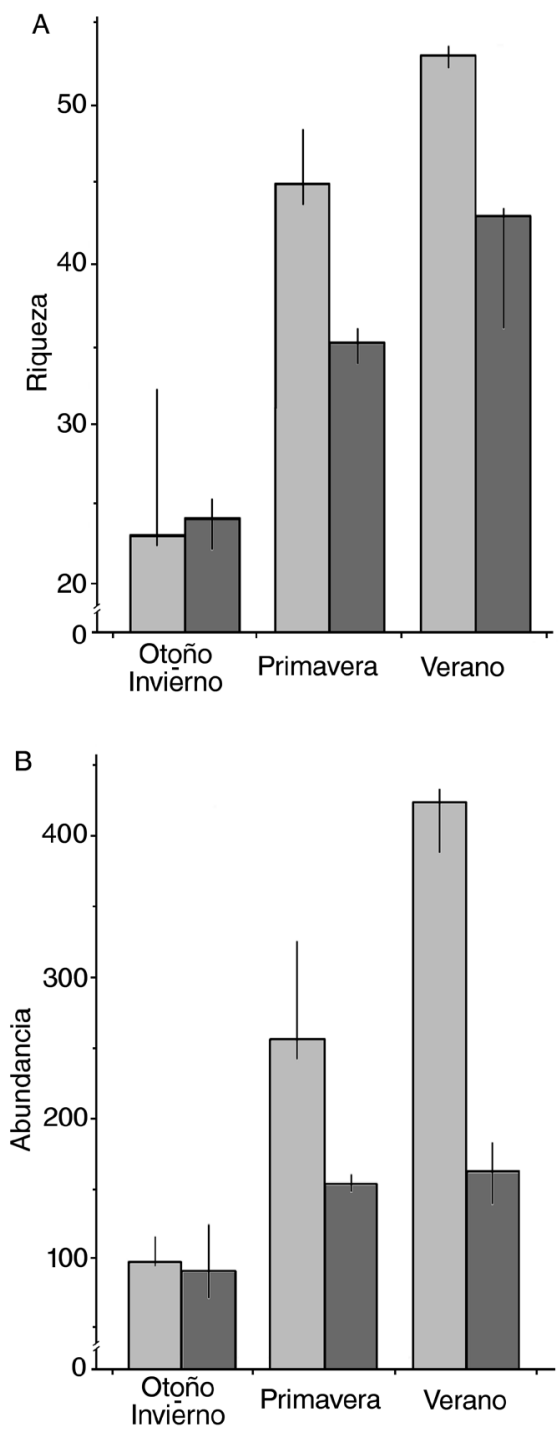

Fig. 1. Variación (A) de la riqueza de especies y (B) de la abundancia de aves (+/-ED) (representada con la media y su error estándar) en función de las estaciones y del hidroperíodo anual (sequía: cajas blancas, lluvioso: caja grises) en el Chaco Serrano Austral, San Juan, Argentina.

Fig. 1. Fluctuations of (A) specimen richness and (B) abundance of birds (represented for the mean and standard error) related to seasonal variations and to annual hydroperiods (dry year: white boxes; rainy year: gray boxes) in the Southern Chaco Serrano, San Juan, Argentina.

Las especies residentes (Fig. 2) fueron las de mayor riqueza (44 especies) y abundancia (2841 individuos), dominadas principalmente por Zonotrichia capensis (Emberizidae), 


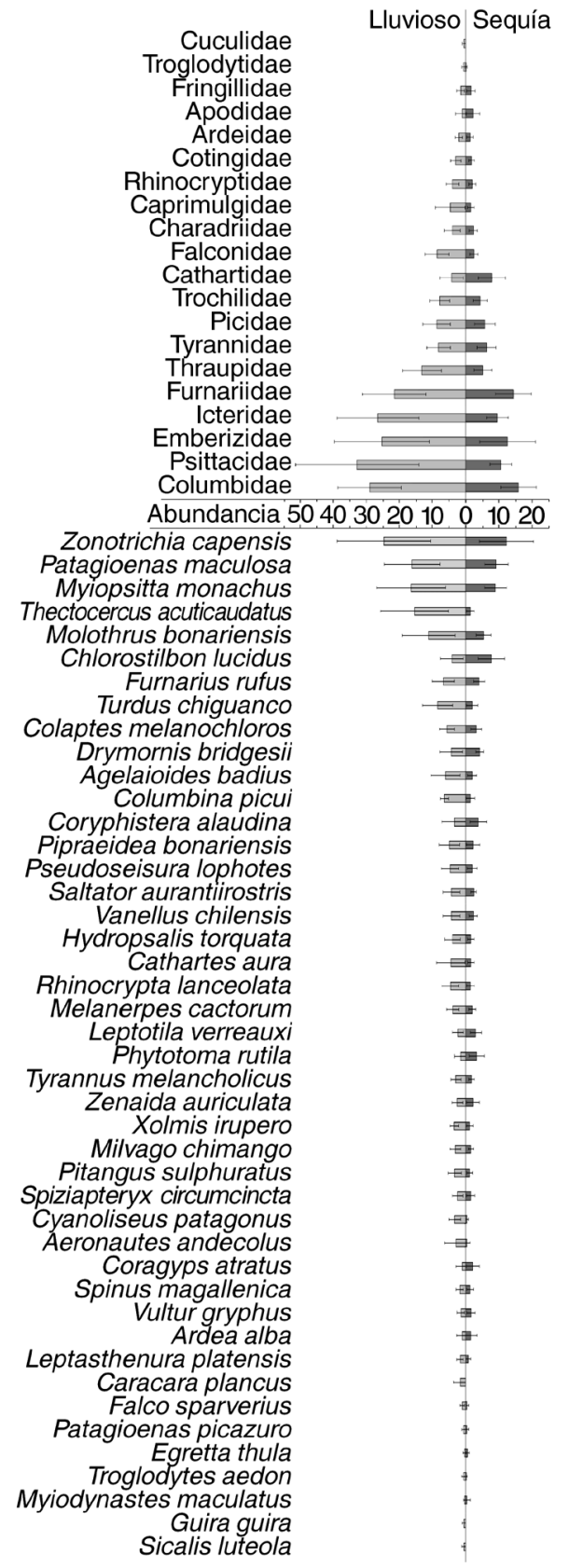

Fig. 2. Abundancia (representada con la media y su error estándar) en las familias y especies de aves residentes, por hidroperíodo anual, en el Chaco Serrano Austral, San Juan, Argentina. La nomenclatura sigue a Remsen et al. (2016). Fig 2. Abundance (represented for the mean and standard error) for the families and species of residents birds, related to annual hydroperiods, in the Southern Chaco Serrano, San Juan, Argentina. The nomenclature follow to Remsen et al. (2016).
Patagioenas maculosa (Columbidae), Myiopsitta monachus, Thectocercus acuticaudatus (ambas Psittacidae) y Molothrus bonariensis (Icteridae) con mayor abundancia durante el año lluvioso y Chlorostilbon lucidus (Trochilidae) durante el año de sequía. Las familias de este grupo presentaron mayor abundancia durante el año lluvioso a excepción de Trochilidae, Apodidae y, con muy baja diferencia, Fringilidae (Fig. 2). Mientras que, las familias mejores representadas en riqueza fueron: Columbidae, Furnaridae (ambas con cinco especies), Thraupidae y Tyrannidae (ambas con cuatro especies).

Las especies estivales tempranas fueron 23 (644 individuos) y fueron mejor representadas en abundancia por Sicalis flaveola (Thraupidae), Synallaxis frontalis (Furnariidae) y Empidonomus aurantioatrocristatus (Tyrannidae) principalmente durante el año lluvioso y Cranioleuca pyrrhophia (Furnariidae) durante el año de sequía (Fig. 3A). Todas las familias de este grupo presentaron mayor abundancia específica en el año lluvioso, representadas principalmente por Furnaridae, Thraupidae y Tyrannidae (Fig. 3A), siguiendo la tendencia de las especies antes mencionadas.

Las especies estivales tardías fueron menos abundantes con seis especies, todas de diferentes familias y mayormente abundantes durante el año lluvioso (Fig. 3B).

Las especies invernales solo fueron representadas por Lessonia rufa (Furnariidae) con mayor abundancia en el año lluvioso y Anairetes parulus (Tyrannidae) con similar abundancia en ambos hidroperiodos. Sin embargo, ninguna de estas dos especies superaron los ocho individuos en los muestreos de mayor abundancia.

También se registró a Columbina talpacoti, Sappho sparganurus, Sporophila caerulescens, Nycticorax nycticorax, Campephilus leucopogon, Asthenes baeri, Agelasticus thilius, Icterus cayanensis, Piranga flava, Nothoprocta cinerascens, Hymenops perspicillatus, Mimus patagonicus, Nothoprocta pentlandii, Ardea cocoi, Geranoaetus polyosoma, Falco peregrinus, Chunga burmeisteri, Glaucidium brasilianum, Asio flammeus, Strix chacoensis, Sublegatus 
A

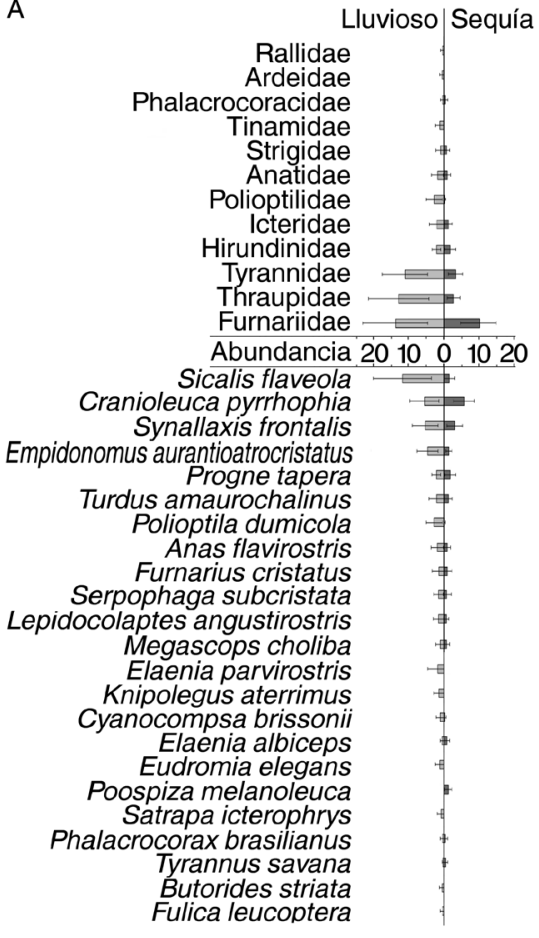

B

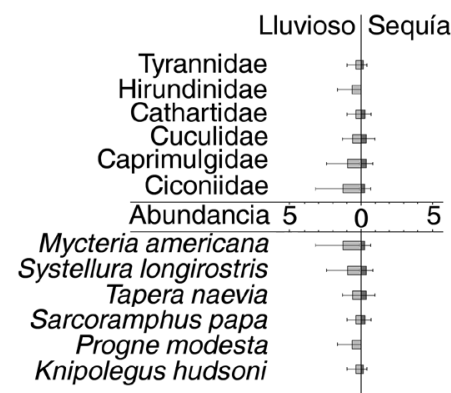

Fig. 3. Abundancia (representada con la media y su error estándar) en las familias y especies de aves (A) estivales tempranas y (B) estivales tardías, por hidroperíodo anual, en el Chaco Serrano Austral, San Juan, Argentina. La nomenclatura sigue a Remsen et al. (2016).

Fig 3. Abundance (represented for the mean and standard error) for the families and species of birds (A) that arrive in spring and (B) that arrive in summer, related to annual hydroperiods, in the Southern Chaco Serrano, San Juan, Argentina. The nomenclature follow to Remsen et al. (2016).

modestus, Muscisaxicola maculirostris y Pyrocephalus rubinus como escasas en abundancia por presentar menos de cuatro individuos en al menos una estación de muestreo.

\section{DISCUSIÓN}

En el sector del Chaco Serrano Austral estudiado, la riqueza de aves quedó representada por el $68 \%$ de las especies citadas en la composición ornitológica general de la región del Chaco (Olrog \& Capllonch, 1986). Asimismo, los registros de las especies encontradas en este estudio presentan una alta similitud (mayor al $90 \%$ ) con la avifauna del Chaco Serrano Austral registrada al sur (Ochoa de Masramón, 1983; Nores \& Yzurieta, 1983; Cid \& Caviedes Vidal, 2005) y al norte del sitio de estudio (Camperi et al., 2008).

Por el contrario, en ambientes que bordean las Sierras de Valle Fértil, donde se presenta un ecotono entre la fitoprovincia del Monte y del Chaco Serrano, Haene et al. (1995) encontraron una menor riqueza específica de aves (32 especies). Si comparamos estos resultados con el presente estudio, existe una baja similitud específica (21\%), aun así, estos autores mencionan que la avifauna tanto en número de especies residentes como migratorias de la región chaqueña, está asociada a la presencia de ciertos ambientes (sierras con arroyos y bosques nativos) que no se encuentran en otras provincias fitogeográficas. Por otra parte, Nores (1995) estudió la avifauna en ambientes puneños y del espinal en el sector sur-oeste del Parque Natural Provincial Valle Fértil, durante un hidroperíodo lluvioso (entre marzo 1990 y febrero 1991, con $510 \mathrm{~mm}$ de precipitación), y la riqueza específica que registró (18 especies) fue baja en similitud $(27 \%)$ con respecto a nuestros resultados. Por lo tanto, se reafirma la hipótesis de que entre ambientes característicos del Chaco Serrano y conservados, como los de este estudio, se esperan valores similares de riqueza y abundancia de aves. Las especies de aves registradas en esta región fitogeográfica presentarían una baja similitud con las de Monte y de Puna, ya que estos últimos ambientes presentan una mayor aridez imperante durante la mayor parte del año, y su flora está compuesta de vegetación xerófila (Márquez et al., 2015). 
Muchas de estas especies de aves se desplazan en un corredor migratorio desde el norte del Chaco Serrano hacia el sur y sudeste de la provincia de San Juan y hacia el norte de San Luis, encontrándose en el borde de su distribución (Chebez \& Gasparri, com. pers.), lo que explicaría que la estacionalidad sea uno de los factores importantes que marca cambios en la avifauna, tanto en su riqueza como en su abundancia. La mayoría de las especies que llegan como visitantes estivales tempranas (primaverales) residen también durante el verano, sumándose en esta última estación las migratorias estivales tardías, lo cual coincide con los resultados encontrados por Codesido y Bilenca (2004) en la avifauna de las sierras subtropicales en la provincia de Córdoba, Argentina. Los pequeños y grandes desplazamientos, el aumento en la actividad diaria requerida para sustentar la demanda energética de los eventos reproductivos (Sibly et al., 2013; de la Peña, 2015), serían los principales factores que permiten encontrar una mayor abundancia en la estación estival temprana. Mientras que, el cambio de plumaje, el incremento poblacional que ocurre inmediatamente después del período de reproducción y su expansión por agotamiento del recurso trófico (Jahn et al., 2006; Buehler \& Piersma, 2008), podrían ayudar a entender el aumento de la riqueza durante la estación de verano y por la llegada de estivales tardías.

Entre los hidroperíodos, el año lluvioso presentó mayor riqueza y abundancia de aves, principalmente en primavera y verano, en concordancia con una estructura vegetal más compleja favorecida por este factor (Brinson, 2004; Maldonado, 2012). Sin embargo, las lluvias mensuales no se asociaron con los aumentos en la abundancia y riqueza de aves. Esto podría deberse a que altas densidades de aves y una mayor interacción entre gremios tróficos es sólo soportada cuando el ambiente proporciona una mayor diversidad de recursos alimenticios (Brinson, 1993).

Es posible que existan otros factores que expliquen una mayor riqueza y abundancia de aves en el área estudiada, como por ejemplo, que el sitio de estudio se encuentre en un área provincial protegida. Sin embargo, en estos ambientes existen zonas impactadas debido a la práctica de motociclismo (enduro), ciclismo, turismo, explotación minera, tala de bosque nativo, captura y venta ilegal de fauna, entre otras actividades de menor impacto, que son consideradas como potenciales modificadores de la comunidad de aves (Isacch, Maceira, Bo, Demaría, \& Peluc, 2005; Codesido, GonzálezFischer, \& Bilenca, 2008).

En síntesis, la avifauna encontrada presenta una elevada similitud con otras áreas del Chaco Serrano donde se intenta preservar su diversidad (Herrera \& Ortiz, 2005; Di Giacomo, et al., 2007), por lo que el área que estudiamos, también debería ser considerada una zona prioritaria para su conservación, principalmente para muchas especies migratorias que llegan antes y durante el periodo reproductivo. La adecuada conservación, es aún un reto tanto para las aves como para sus hábitats y la tarea se dificulta más si se tratan las variables que las afectan de forma aislada (Williams, de Klerk, \& Crow, 1999; Kark \& Van Rensburg, 2006). De este modo, las variaciones de la riqueza y la abundancia de aves del Chaco Serrano Austral observadas entre las diferentes estaciones e hidroperíodos anuales (excepto las lluvias mensuales), contribuirán con las tareas de manejo y conservación.

\section{AGRADECIMIENTOS}

Agradecemos especialmente a Daisy Arroyo Mora y a los revisores anónimos que con sus aportes mejoraron la versión inicial. A Rodrigo Acosta, Melina Rodriguez, Rodrigo Nievas, Lía Piaggio, Susana Castro, Matías Olmedo por su ayuda y compañía en el campo y a Tomas Martinez quien también realizó algunas sugerencias.

\section{RESUMEN}

En las regiones subtropicales, las precipitaciones tienen una marcada estacionalidad. El agua de inundaciones causadas por fuertes lluvias puede tener consecuencias importantes para los ecosistemas dependientes. Por el contrario, en periodos de sequía algunas especies de la fauna se encuentran limitadas a la oferta de los recursos disponibles en el hábitat, reflejando cambios 
en su diversidad y distribución. Entre los vertebrados, las aves son consideradas como excelentes indicadores ecológicos y facilitan evaluar los cambios de su hábitat. Sin embargo, en el Chaco Serrano Austral argentino poco se conoce sobre la avifauna. Nosotros estudiamos (1) el estado de residencia que presentan las especies de aves en el área austral del Chaco Serrano y (2) la relación de su riqueza y abundancia con la variación estacional y con los efectos de hidroperíodos mensuales y anuales. Realizamos 18 muestreos durante un período de dos años (marzo 2011 - febrero 2013) en el sector noreste del Parque Natural Valle Fértil en la provincia de San Juan, Argentina. Estos muestreos se llevaron a cabo durante los períodos no reproductivo (otoño-invierno), pre-reproductivo (primavera) y reproductivo (verano), con el fin de evaluar el estado de residencia de las aves. En cada muestreo se registraron las poblaciones de aves utilizando cinco transectas, con el fin de registrar su abundancia y riqueza específica. Con el fin de analizar las relaciones entre las variables se utilizó un modelo lineal generalizado mixto. Se registraron 98 especies de aves (44 especies residentes, 23 visitantes que llegan en primavera, seis visitantes de verano, dos visitantes de invierno y 23 con registros escasos), pertenecientes a 33 familias. Asimismo, registramos 68 especies en ambos hidroperíodos, 28 durante el hidroperíodo de lluvia y dos durante el hidroperíodo de sequía. Tanto la riqueza, como la abundancia de aves fueron mayores en el periodo prereproductivo y durante el hidroperíodo anual lluvioso, mientras que las lluvias mensuales aisladas no presentaron esta tendencia. La avifauna registrada en este artículo es similar a la encontrada en las regiones del Chaco Serrano, que fue identificado como una región clave para la preservación de la biodiversidad. Por lo tanto, recomendamos tomar esta área como prioritaria para la conservación. Los cambios en la abundancia de aves se asociaron con la llegada de las especies migratorias, sobre todo con las visitantes estivales tempranas, y probablemente en relación con su actividad reproductiva y la evolución estacional de los recursos alimentarios. El Chaco Serrano Austral subtropical semiárido parece ser un importante lugar de cría para muchas especies migratorias que además promueven los cambios estacionales en la densidad y diversidad de especies, sobre todo en los años lluviosos. La creciente comprensión en las causas de variación de la riqueza y abundancia de aves del Chaco Serrano Austral observadas en este trabajo, contribuirán con las tareas manejo y conservación de este importante grupo faunístico.

Palabras claves: ornitología, Parque Provincial Natural Valle Fértil, ecología, hidroperíodo, sequía, semiárido.

\section{REFERENCIAS}

Bates, D., Maechler, M., Bolker, B., \& Walker, S. (2015). lme4: Linear mixed-effects models using Eigen and S4. $R$ package version, $1(9)$.
Beltzer, A. H., \& Neiff., J. J. (1992). Distribución de las aves en el valle del río Paraná. Relación con el régimen pulsátil y la vegetación. Ambiente Subtropical, $2,77-102$.

Bibby, C., Jones, M., \& Marsden, S. (1998). Bird surveys, expedition field techniques. London, U.K.: Expedition Advisory Center, Royal Geographical Society.

Bolker, B. M., Brooks, M. E., Clark, C. J., Geange, S. W., Poulsen, J. R., Stevens, M. H., \& White, J. S. (2009). Generalized linear mixed models: a practical guide for ecology and evolution. Trends in Ecology \& Evolution, 24, 127-135.

Brinson, M. M. (1993). A hydrogeomorphic classification for wetlands. Wetlands Research Program Technical Report WRP-DE-4. Vicksburg, MS, U.S.: US Army Corps of Engineers Waterways Experiment Station.

Brinson, M. M. (2004). Niveles extremos de variación de patrones y procesos en humedales. En A. I. Malvárez \& R. F. Bó (Eds.), Bases ecológicas para la clasificación e inventario de humedales en Argentina (pp. 19-24). Buenos Aires: FCEN (UBA)-RAMSAR-USFWS-USDS.

Buehler, D. M., \& Piersma, T. (2008). Travelling on a budget: predictions and ecological evidence for bottlenecks in the annual cycle of long-distance migrants. Philosophical Transactions of Royal Society $B, 363,247-266$.

Camperi, A. R., Darrieu, C. A., \& Juárez, M. (2008). Avifauna de la provincia de La Rioja (Argentina): lista comentada de especies. Acta Zoológica Lilloana, 52(1-2), 76-97.

Capurro, H. A., \& Bucher, E. H. (1988). Lista comentada de las aves del Bosque Chaquefio de Joaquin V. Gonzilez, Salta, Argentina. Hornero, 13, 39-46.

Casco, S. L., Neiff, M., \& Neiff, J. J. (2005). Biodiversidad en ríos del litoral fluvial. Utilidad del software PULSO. Temas de la Biodiversidad del Litoral fluvial argentino II. Miscelánea, 14, 419434.

Cid, F. D., \& Caviedes Vidal, E. (2005). Avifauna del Embalse La Florida (San Luis, Argentina). Actualidades Ornitológicas, 125, 10-18.

Codesido, M., \& Bilenca, D. (2004). Variación Estacional de un Ensamble de Aves en un Bosque Subtropical Semiárido del Chaco Argentino. Biotrópica, 36(4), 544-554.

Codesido, M., González-Fischer, C., \& Bilenca, D. (2008). Asociaciones entre diferentes patrones de uso de la tierra y ensambles de aves en agroecosistemas de la Región Pampeana, Argentina. Ornitología Neotropical, 19, 575-585.

de la Peña, M. R. (2015). Aves argentinas, incluye nidos y huevos. Tomo 1 y 2. Buenos Aires: Eudeba. 
Di Giacomo, A. S., De Francesco, M. V., \& Coconier, E. G. (2007). Áreas importantes para la conservación de las aves en Argentina. Sitios prioritarios para la conservación de la biodiversidad. Temas de Naturaleza y Conservación 5. Buenos Aires: Aves Argentinas/ Asociación Ornitológica del Plata.

Ginzburg, R., Adámoli, J., Herrera, P., \& Torrella, S. (2005). Los Humedales del Chaco: clasificación, inventario y mapeo a escala regional. Miscelánea, 14, 121-138.

Haene, E. H., Krapovickas, S. F., Moschione, F., \& Gómez, D. (1995). Observaciones y comentarios biogeográficos sobre la avifauna del este de la provincia de San Juan, Argentina. Hornero, 14, 48-52.

Herrera, P., \& Ortiz, U. M. (2005). La evaluación ecorregional del Gran Chaco Americano. Identificación de las áreas más importantes para la conservación. En A. Brown, U. M. Ortiz, M. Acerbi, \& J. Corcuera (Eds.), La Situación Ambiental Argentina 2005, Ecorregión Chaco Seco (pp. 94-100). Buenos Aires: Fundación Vida Silvestre Argentina.

Huxman, T. E., Wilcox, B. P., Breshears, D. D., Scott, R. L., Snyder, K. A., Small, E. E., Hultine, K., Pockman, W. T., \& Jackson, R. B. (2005). Ecohydrological implications of woody plant encroachment. Ecology, 86, 308-319.

Isacch, J. P., Maceira, N. O., Bo, M. S., Demaría, M. R., \& Peluc, S. (2005). Bird-habitat relationship in semiarid natural grasslands and exotic pastures in the west pampas of Argentina. Journal of Arid Environments, $62,267-283$.

Jahn, A. E., Levey, D. J., Johnson, J. E., Mamani, A. M., \& Davis, S. E. (2006). Towards a mechanistic interpretation of bird migration in South America. Hornero, 21(02), 099-108

Kark, S., \& Van Rensburg, B. J. (2006). Ecotones: Marginal or Central Areas of Transition? Israel Journal of Ecology \& Evolution, 52(1), 29-53.

Lourenço, P. M., \& Piersma, T. (2015). Migration distance and breeding latitude correlate with the scheduling of pre-alternate body moult: a comparison among migratory waders. Journal of Ornithology, $156(3), 657-665$

Maldonado, L. J. (2012). Caracterización y usos de los recursos naturales en zonas áridas. Revista Mexicana de Ciencias Forestales, 4(20), 56-63.

Marateo, G., \& Arturi, M. (2013). Dinámica estacional y variación local de gremios tróficos de aves de una selva en galería y un palmar subtropical de sudamérica. Ornitologia Neotropical, 24, 213-223.
Márquez, J., Ripoll, Y., Dalmaso, A., Ariza, M., \& Jordan, M. (2015). Árboles Nativos de la Provincia de San Juan. San Juan, Argentina: Universidad Nacional de San Juan.

Milesi, F. A., Marone, L., Lopez de Casenave, J., Cueto, V. R., \& Mezquida, E. T. (2002). Gremios de manejo como indicadores de las condiciones del ambiente: un estudio de caso con aves y perturbaciones del hábitat en el Monte central, Argentina. Ecología Austral, $12,149-161$.

Narosky, T., \& Yzurieta, D. (2010). Guía para la identificación de las aves de Argentina y Uruguay. Edición de Oro. Buenos Aires: Vázquez Mazzini Editores.

Nores, M. (1995). Insular biogeography of birds on montain-tops in north western Argentina. Journal of Biogeography, 22, 61-70.

Nores, M., \& Yzurieta, D. (1983). Especiación en las Sierras Pampeanas de Córdoba y San Luis (Argentina), con descripción de siete nuevas subespecies de aves. Hornero, 12, 088-102.

Ochoa de Masramón, D. (1983). Lista de aves del nordeste de San Luis. Hornero, 12, 077-087.

Olrog, C. C., \& Capllonch, P. (1986). Bio-ornitología Argentina. Historia natural, Suplemento, 2.

Remsen, J. V., Areta, J. I., Cadena, C. D., Claramunt, S., Jaramillo, A., Pacheco, J. F., Pérez-Emán, J., Robbins, M. B., Stiles, F. G., Stotz, D. F., \& Zimmer, K. J. (2016). A classification of the bird species of South America. American Ornithologists' Union. [20 September 2016]. Recuperado de http://www.museum. 1su.edu/ Remsen/SACCBaseline.htm

Sarmiento, G. (1972). Ecological and floristic convergence between seasonal plant formations of Tropical and Subtropical South America. Ecology, 60, 367-410.

Sibly, R. M., Grimm, V., Martin, B. T., Johnston, A. S. A., Topping, C. J., Kułakowska, K., Topping, C. J., Calow, P., Nabel-Nielsen, J., Thorbek, P., \& DeAngelis, D. L. (2013). Representing the acquisition and use of energy by individuals in agent-based models of animal populations. Methods in Ecology and Evolution, 4, 151-161.

The R Development Core Team (2015). R: A language and environment for statistical computing. Massachusetts: R Foundation Project-GNU project.

Williams, P. H., de Klerk, H. M., \& Crow, T. M. (1999). Interpreting biogeographical boundaries among Afrotropical birds: spatial patterns in richness gradients and species replacement. Journal of Biogeography, $26,459-474$. 